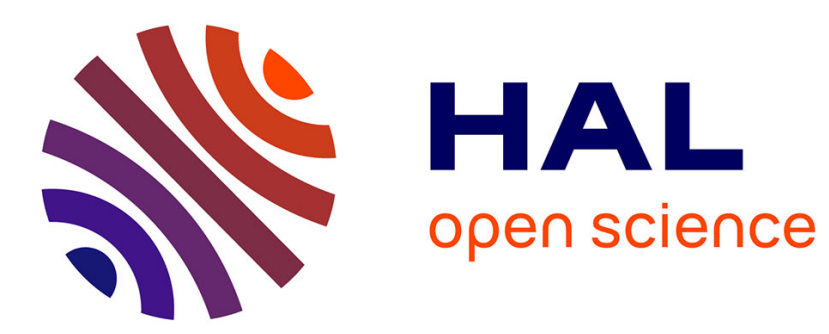

\title{
Incorporating the modified orifice equation into pipe network solvers for more realistic leakage modeling
}

Asaph Mercy Kabaasha, Olivier Piller, Jakobus Ernst van Zyl

\section{To cite this version:}

Asaph Mercy Kabaasha, Olivier Piller, Jakobus Ernst van Zyl. Incorporating the modified orifice equation into pipe network solvers for more realistic leakage modeling. Journal of Hydraulic Engineering, 2018, 144 (2), 04017064, 8 p. 10.1061/(ASCE)HY.1943-7900.0001410 . hal-01764568

\section{HAL Id: hal-01764568 \\ https://hal.science/hal-01764568}

Submitted on 12 Apr 2018

HAL is a multi-disciplinary open access archive for the deposit and dissemination of scientific research documents, whether they are published or not. The documents may come from teaching and research institutions in France or abroad, or from public or private research centers.
L'archive ouverte pluridisciplinaire $\mathbf{H A L}$, est destinée au dépôt et à la diffusion de documents scientifiques de niveau recherche, publiés ou non, émanant des établissements d'enseignement et de recherche français ou étrangers, des laboratoires publics ou privés. 
Author-produced version of the article published in J. Hydraul. Eng. (ASCE), 144(2); doi:10.1061/(ASCE)HY.1943-7900.0001410. The original publication is available at https://ascelibrary.org/doi/abs/10.1061/\%28ASCE\%29HY.1943-7900.0001410;

INCORPORATING THE MODIFIED ORIFICE EQUATION INTO PIPE NETWORK SOLVERS FOR MORE REALISTIC LEAKAGE

\title{
MODELING
}

\author{
A.M. Kabaasha ${ }^{1}$, O. Piller ${ }^{2}$, J.E. van Zyl ${ }^{3}$ \\ ${ }^{1}$ Department of Civil Engineering, University of Cape Town, Cape Town, Rondebosch 7701, South \\ Africa. \\ ${ }^{2}$ Irstea, UR ETBX, Water Department, Bordeaux Regional Centre, Cestas F-33612, France. \\ ${ }^{3}$ Department of Civil Engineering, University of Cape Town, Cape Town, Rondebosch 7701, South \\ Africa. \\ Correspondence to: A.M. Kabaasha (KBSASA001@myuct.ac.za)
}

\section{KEYWORDS}

Modified orifice equation; leakage modeling; pressure management; water distribution systems

\begin{abstract}
It has been well established in several experimental and modeling studies that leak areas are often not fixed, but vary as linear functions of pressure. Replacing this linear equation into the orifice equation results in a two-part modified orifice leakage equation with head exponents of 0.5 and 1.5 respectively. The purpose of this study was to incorporate the modified orifice equation into the hydraulic network formulation and evaluate its impact on model performance. The conventional and modified software were applied to 600 instances of stochastic leakage distributions in three different pipe networks. It was found that the conventional power leakage equation results in significant leakage volume and flow rate errors under certain conditions. In addition, a problem of non-convergence of the conventional global
\end{abstract}


gradient algorithm for leakage exponents greater than two was observed and is discussed.

\section{INTRODUCTION}

Hydraulic network modeling software, including the commonly used Epanet package, (Rossman, 2000), employs a power equation to model pressure dependent outflows such as leakage. Researchers and practitioners in the leakage field have long realized that leakage does not adhere to the theoretical orifice equation (a power equation with a fixed exponent of 0.5 ) but mostly require higher power exponents to be simulated realistically (Ogura, 1979; Hiki, 1981).

Several causes for the power leakage exponent diverging from the theoretical value of 0.5 have been investigated, including leakage hydraulics (Van Zyl \& Clayton, 2007), soil-leak interaction (Walski, 2006; Van Zyl et al., 2013) and the distribution of leaks in a network (Schwaller \& Van Zyl, 2014). However, the overriding cause of variations in the leakage exponent has been shown to be that leak areas are not fixed, but vary with system pressure (May, 1994, Van Zyl \& Clayton, 2007; Cassa et al., 2010; Ferrante, et al., 2011; Massari et al, 2012; De Marchis et al., 2016; Fox et al, 2016). In addition, the variations in leak area have been shown to be a linear function of pressure head under both elastic and viscoelastic deformation conditions for different leak types, pipe materials and loading states (Cassa \& Van Zyl, 2013; Van Zyl \& Cassa, 2014; Malde, 2015; Ssozi et al, 2016).

The variations in leak area under plastic deformation and fracture conditions cannot be assumed linear, but Van Zyl et al (in press) argues that these mechanisms are unlikely to dominate the pressure-leakage response of distribution systems because they a) are irreversible and b) only occur when pressures are increased and not when 
they are decreased. Thus, it is assumed in this study that all leak areas vary linearly with pressure head.

Van Zyl et al (in press) investigated the implications of a linear head-area relationship and concluded that the power equation is flawed in that its parameters aren't constant, but vary with system pressure, and that the leakage exponent approaches infinity under certain conditions. They concluded that significant modeling errors are possible if the power equation is extrapolated beyond its calibration pressure range and at high exponent values. Finally, they recommended that the linear head-area relationship be explicitly incorporated in hydraulic simulation software for more realistic leakage modeling.

The aim of this study was to incorporate and test the inclusion of a modified orifice equation, which explicitly includes a linear head-area relationship in the standard hydraulic network solver model.

The next section develops a modified orifice equation that incorporates a linear pressure-area relationship, describes its relationship to the power formulation and provides a brief description of relevant water loss benchmarks. The standard hydraulic network model is then modified to incorporate the linear head-area relationship. Finally, the implications of this modification for model convergence and accuracy are investigated based on three example networks with stochastic leak distributions.

\section{BACKGROUND}

\section{Realistic leakage modeling}

Hydraulically, leaks are orifices that should adhere to the orifice equation:

$$
Q=C_{d} A \sqrt{2 g h}
$$


Where $Q$ is the orifice flow rate, $C_{d}$ discharge coefficient, $A$ orifice area, $g$ acceleration due to gravity and $h$ the head differential over the leak.

The areas of real leaks are not constant, but can be assumed to vary linearly with pressure, i.e.:

$$
A=A_{0}+m h
$$

Where $A_{0}$ is the initial area (the area of the leak opening when the head differential is zero) and $m$ the head-area slope.

Replacing (2) into (1) results in the modified orifice equation, known in leakage practice as the FAVAD (Fixed and Variable Area Discharges) equation (May, 1994):

$$
Q=C_{d} \sqrt{2 g}\left(A_{0} h^{0.5}+m h^{1.5}\right)
$$

An important consequence of the linearity assumption of the head-area relationship is that the total leakage area of a system with many leaks will also display a linear headarea relationship. In addition, the total system initial area $\left(A_{0}\right)$ will be the sum of all the individual leak initial areas, and the total system head-area slope $(m)$ will be the sum of all the individual leak head-area slopes (Schwaller et al., 2015). This means that Eq. (3) may be applied equally to a system (or pipe) with a single or multiple leaks.

A power formulation is commonly used to model the behavior of leaks, both in hydraulic modeling software (Rossman, 2000) and leakage management practice (Lambert, 2000; Farley and Trow, 2003):

$$
Q=C h^{\alpha}
$$


Where $C$ is the leakage coefficient and $\alpha$ the leakage exponent. In modeling practice, such as the widely used Epanet software, the power equation is called an emitter (Rossman, 2000) and is used to model pressure-dependent consumer demands and leakage. In leakage management practice, Eq. (4) is called the power leakage equation and the symbol N1 is used instead of $\alpha$ (Lambert, 2000).

\section{Leakage benchmarks}

A brief overview is provided of internationally accepted water loss terminology and benchmarks relevant to this study (AWWA, 2016). It is desirable to split a large distribution system into smaller discrete zones called DMAs (district metered areas). Ideally DMAs should be supplied through a single metered supply point where, if desired, the inlet pressure can be controlled.

The level of real losses in a DMA is appraised using the concept of an infrastructure leakage index (ILI), defined by:

$$
I L I=\frac{C A R L}{U A R L}
$$

Where CARL is the current annual real losses and UARL the unavoidable annual real losses in the system. The CARL is normally estimated by measuring the minimum night flow rate (MNF) entering a DMA (typically between 3:00 and 5:00 in the morning) and subtracting the estimated user consumption.

The UARL (in L/day) represents the theoretical low limit of leakage that could be achieved in a specific system at a given pressure (AWWA, 2016; Lambert, 2009). The UARL formula is given by:

$$
U A R L=\left(18 L_{m}+0.8 N_{c}+25 L_{s}\right) h_{A Z}
$$


Where $L_{m}$ is the length of mains (in $\mathrm{km}$ ), $N_{c}$ the number of service connections, $L_{s}$ the length of service lines between the property boundary and water meter (in $\mathrm{km}$ ) and $h_{A Z}$ the average zonal pressure head. The length of service lines $L_{s}$ is only included in Eq. (6) when consumer meters are installed within the property boundary, otherwise it is set to zero.

\section{MODEL DEVELOPMENT}

The aim of this section is to propose a hydraulic model formulation that incorporates the modified orifice equation (3) for leakage modeling. The standard model formulation is first presented and the modifications to implement the modified orifice equation are then discussed.

\section{Network formulation with standard leakage power function}

The topology of a water distribution network is represented by a set of nodes and links (a graph). A link corresponds to a collection of pipes, valves and pumps.

The steady state system equations are formulated by applying principles of conservation of mass and energy at each node and link respectively. These equations are solved simultaneously to obtain pressure heads at the junction nodes and flow rates in the links.

User demands are lumped at network nodes and consist of both fixed and pressuredependent demands. The standard model for pressure-dependent demands and leakage is a power function:

$$
\mu_{i}=c_{i}\left(H_{i}-z_{i}\right)^{\alpha}, \text { if } H_{i} \geq z_{i}
$$

Where $\mu_{\mathrm{i}}$ is the lumped nodal leakage outflow, $c_{i}$ the leakage coefficient, $z_{i}$ the elevation of node $i$ and $\alpha$ the leakage exponent. 
The steady state equations read:

$$
\begin{aligned}
& -\sum_{j=1}^{n p} A_{i j} Q_{j}-\mu_{i}=D_{i}, \quad i=1, \cdots, n u \\
& h_{j}\left(Q_{j}\right)-\sum_{i=1}^{n u} A_{i j} H_{i}=\sum_{i=1}^{n f} B_{i j} H_{i}^{f}, j=1, \cdots, n p \\
& c_{i}{ }^{-\frac{1}{\alpha}}\left\lfloor\mu_{i}\right\rfloor^{\frac{1}{\alpha}-1} \mu_{i}+h_{i}^{C V}\left(\mu_{i}\right)-H_{i}=-z_{i}, \quad i=1, \cdots, n u
\end{aligned}
$$

Where $n u$ is the number of junction nodes; $n f$ the number of fixed-head nodes; $n p$ the number of links; $Q_{j}$ the flow rate in link $j ; D_{i}$ the fixed demand at node $i ; A_{i j}$ a coefficient describing the existence and direction of link $j$ (-1 if link $j$ is directed toward node $i,+1$ if link $j$ is directed away from node $i$, and 0 if link $j$ is not connected to node $i$ ); $B_{i j}$ is similar to $A_{i j}$ but for fixed-head nodes; $H_{i}$ is the head at node $i$; $H_{i}^{f}$ the head at fixed-head node $i ; h_{j}$ the headloss in link $j$, and $h_{i}^{C V}$ the check valve head loss penalty function as proposed in Piller and van Zyl (2014) and given by:

$$
h_{i}^{C V}\left(\mu_{i}\right)=-r_{\max } \max \left(0,-\mu_{i}\right)^{2}=-h_{0} \max \left(0,-\frac{\mu_{i}}{\Delta Q}\right)^{2}
$$

Where $r_{\max }=\frac{h_{0}}{\Delta Q^{2}}$ is a large resistance coefficient, which is chosen to produce an equivalent head loss less than or equal to $-h_{0}$ if the leakage outflow is less than or equal $-\Delta Q$; for practical application, $h_{0}=15 \mathrm{~m}$ and $\Delta Q=0.01 \mathrm{~L} / \mathrm{s}$.

In Epanet, emitters at nodes are modeled by adding a fictitious pipe between the node and a fictitious reservoir (Rossman, 2000). The head at the fictitious reservoir is the elevation of the junction. The frictional pipe head loss is described using Eq. (7), with resistance $c^{-1 / \alpha}$ and exponent $1 / \alpha$. The last equation in system shown in Eq. (8) describes the behavior of the fictional pipe and reservoir and, unlike the Epanet emitter function, includes a check valve to prevent backflow. 
The function $h_{j}$ is differentiable with respect to $Q_{j}$. Moreover, we assume $h_{j}$ is a strictly increasing function of $Q_{j}$, which is non-bounded at infinity. The system shown in Eq. (8) can be rewritten in matrix form as:

$$
\begin{gathered}
-\mathbf{A Q}-\boldsymbol{\mu}=\mathbf{D} \\
\mathbf{h}(\mathbf{Q})-\mathbf{A}^{T} \mathbf{H}=\mathbf{B}^{T} \mathbf{H}^{f} \\
\mathbf{g}(\boldsymbol{\mu})-\mathbf{H}=-\mathbf{Z}
\end{gathered}
$$

Where $\mathbf{Q}$ is a vector of link flow rates; $\mathbf{D}$ a vector of fixed nodal demands; $\mathbf{A}$ an $n u \mathbf{x}$ $n p$ incidence matrix of the variable-head nodes, $\mathbf{B}$ an $n f \times n p$ incidence matrix of fixed-head nodes; $\mathbf{H}$ a vector of variable nodal heads, $\mathbf{H}^{\mathrm{f}}$ a vector of fixed nodal heads; $\mathbf{h}$ a vector of link head losses; $\mathbf{Z}$ a vector of elevations at variable-head nodes; and $\mathbf{g}(\boldsymbol{\mu})$ a vector of link head losses in the fictional pipes.

Eq. (10) has one and only one solution if matrix $\mathbf{A}$ is full rank in the number of junction nodes (Piller, 1995). Indeed, there exists an elliptic function (the Content function) whose minimization restricted to the mass balance equation is equivalent to solving Eq. (10). For $\mathbf{A}$ to be full rank, it is sufficient that each connected component or group of nodes possesses at least one fixed-head node.

The method of Newton applied to system (10) is an iterative procedure that consists of sequentially solving the linear system:

$$
\left(\begin{array}{ccc}
-\mathbf{A} & -\mathbf{I}_{n u, n u} & \mathbf{0}_{n u, n u} \\
\mathbf{J}_{n} & \mathbf{0}_{n p, n u} & -\mathbf{A}^{T} \\
\mathbf{0}_{n u, n p} & \mathbf{K}_{n} & -\mathbf{I}_{n u, n u}
\end{array}\right)\left(\begin{array}{c}
\mathbf{Q}^{n+1}-\mathbf{Q}^{n} \\
\boldsymbol{\mu}^{n+1}-\boldsymbol{\mu}^{n} \\
\mathbf{H}^{n+1}-\mathbf{H}^{n}
\end{array}\right)=\left(\begin{array}{c}
\mathbf{A} \mathbf{Q}^{n}+\boldsymbol{\mu}^{n}+\mathbf{D} \\
-\mathbf{h}\left(\mathbf{Q}^{n}\right)+\mathbf{A}^{T} \mathbf{H}^{n}+\mathbf{B}^{T} \mathbf{H}^{f} \\
-\boldsymbol{g}\left(\boldsymbol{\mu}^{n}\right)+\mathbf{H}^{n}-\mathbf{Z}
\end{array}\right)
$$

Where $\mathbf{J}_{n}=\nabla_{\mathbf{Q}} \mathbf{h}\left(\mathbf{Q}^{n}\right)$ is the Jacobian matrix of the $\mathbf{h}$ function with regards to $\mathbf{Q}$ at $\mathbf{Q}^{\boldsymbol{n}}$; and $\mathbf{K}_{n}=\nabla_{\boldsymbol{\mu}} \mathbf{g}\left(\boldsymbol{\mu}^{\mathbf{n}}\right)$ is the Jacobian matrix of the $\mathbf{g}$ function with regards to $\boldsymbol{\mu}$ at $\mu^{n}$ 
It may be observed that $\mathbf{Q}^{\mathrm{n}+1}$ satisfies the mass-balance equation, as the first row of Eq. (11) reduced to $-\mathbf{A} \mathbf{Q}^{n+1}-\boldsymbol{\mu}^{n+1}=\mathbf{D}$.

Eq. (11) may be further reduced by applying a block Gaussian elimination to the system

$$
\begin{gathered}
\mathbf{Q}^{n+1}=\mathbf{Q}^{n}-\mathbf{J}_{n}{ }^{-1}\left[\mathbf{h}\left(\mathbf{Q}^{n}\right)-\mathbf{A}^{T} \mathbf{H}^{n+1}-\mathbf{B}^{T} \mathbf{H}^{f}\right] \\
\boldsymbol{\mu}^{n+1}=\boldsymbol{\mu}^{n}-\mathbf{K}_{n}{ }^{-1}\left[\boldsymbol{g}\left(\boldsymbol{\mu}^{n}\right)-\mathbf{H}^{n+1}+\mathbf{Z}\right]
\end{gathered}
$$

Where $\mathbf{H}^{\mathrm{n}+1}$ is solution of the linear system:

$$
\begin{gathered}
\left(\mathbf{A} \mathbf{J}_{n}{ }^{-1} \mathbf{A}^{T}+\mathbf{K}_{n}{ }^{-1}\right) \mathbf{H}^{n+1}=\cdots \\
\mathbf{A J}_{n}{ }^{-1}\left[\mathbf{h}\left(\mathbf{Q}^{n}\right)-\mathbf{B}^{T} \mathbf{H}^{f}\right]+\mathbf{K}_{n}{ }^{-1}\left[\boldsymbol{g}\left(\boldsymbol{\mu}^{n}\right)+\mathbf{Z}\right]-\left[\mathbf{A Q}^{n}+\boldsymbol{\mu}^{n}+\mathbf{D}\right]
\end{gathered}
$$

Equations (12) and (13) are the foundation for the so-called hybrid methods (Hamam \& Brameller, 1971; Carpentier et al., 1985; Todini and Pilati, 1988). One implementation of these hybrid methods is the global gradient algorithm (GGA) used in Epanet, which follows from the application of the Newton method to the system Eq. (10).

\section{Modified orifice formulation}

The modified orifice formulation has two important differences to the power equation: it requires the addition of two fictional pipe-reservoir systems to each node to simulate the two terms in (3) and the exponents of the two terms are fixed at 0.5 and 1.5 .

The modified orifice equation (3) is written as follows:

$$
\mu_{i}=\mu_{1 i}+\mu_{2 i}=c_{1 i}\left(H_{i}-z_{i}\right)^{0.5}+c_{2 i}\left(H_{i}-z_{i}\right)^{1.5}, \text { if } H_{i} \geq z_{i}
$$

Where $\mu_{1 i}$ is the lumped leakage outflow corresponding to exponent $0.5 ; \mu_{2 i}$ is the lumped leakage outflow corresponding to exponent 1.5 ; coefficient $c_{1 i}$ is equal to the 
sum of $\sqrt{2 g} C_{d} A_{0}$, and $c_{2 i}$ is equal to the sum of $\sqrt{2 g} C_{d} m$ of all the individual leaks lumped to node $i$.

Including the modified orifice equation in the initial system in Eq. (8) leads to:

$$
\begin{gathered}
-\sum_{j=1}^{n p} A_{i j} Q_{j}-\mu_{1 i}-\mu_{2 i}=D_{i}, \quad i=1, \cdots, n u \\
h_{j}\left(Q_{j}\right)-\sum_{i=1}^{n u} A_{i j} H_{i}=\sum_{i=1}^{n f} B_{i j} H_{i}^{f}, \quad j=1, \cdots, n p \\
c_{1 i}{ }^{-2}\left\lfloor\mu_{1 i}\right\rfloor \mu_{1 i}+h_{i}^{C V}\left(\mu_{1 i}\right)-H_{i}=-z_{i}, \quad i=1, \cdots, n u \\
c_{2 i}{ }^{-\frac{2}{3}}\left\lfloor\mu_{2 i}\right\rfloor^{-\frac{1}{3}} \mu_{2 i}+h_{i}^{C V}\left(\mu_{2 i}\right)-H_{i}=-z_{i}, \quad i=1, \cdots, n u
\end{gathered}
$$

The equivalent matrix form is:

$$
\begin{gathered}
-\mathbf{A Q}-\boldsymbol{\mu}_{1}-\boldsymbol{\mu}_{2}=\mathbf{D} \\
\mathbf{h}(\mathbf{Q})-\mathbf{A}^{T} \mathbf{H}=\mathbf{B}^{T} \mathbf{H}^{f} \\
\mathbf{g}^{F}\left(\boldsymbol{\mu}_{1}\right)-\mathbf{H}=-\mathbf{Z} \\
\mathbf{g}^{\mathrm{V}}\left(\boldsymbol{\mu}_{2}\right)-\mathbf{H}=-\mathbf{Z}
\end{gathered}
$$

Where $\mathbf{g}^{F}\left(\boldsymbol{\mu}_{1}\right)$ is a vector of link head losses at fixed-area fictional pipes; and $\mathbf{g}^{V}\left(\boldsymbol{\mu}_{2}\right)$ a vector of link head losses at variable-area fictional pipes.

The method of Newton applied to system (16) consists of sequentially solving the linear system:

$$
\left(\begin{array}{cccc}
-\mathbf{A} & -\mathbf{I}_{n u, n u} & -\mathbf{I}_{n u, n u} & \mathbf{0}_{n u, n u} \\
\mathbf{J}_{n} & \mathbf{0}_{n p, n u} & \mathbf{0}_{n p, n u} & -\mathbf{A}^{T} \\
\mathbf{0}_{n u, n p} & \mathbf{F}_{n} & \mathbf{0}_{n u, n u} & -\mathbf{I}_{n u, n u} \\
\mathbf{0}_{n u, n p} & \mathbf{0}_{n u, n u} & \mathbf{V}_{n} & -\mathbf{I}_{n u, n u}
\end{array}\right)\left(\begin{array}{c}
\mathbf{Q}^{n+1}-\mathbf{Q}^{n} \\
\boldsymbol{\mu}_{1}{ }^{n+1}-\boldsymbol{\mu}_{1}{ }^{n} \\
\boldsymbol{\mu}_{2}{ }^{n+1}-\boldsymbol{\mu}_{2}{ }^{n} \\
\mathbf{H}^{n+1}-\mathbf{H}^{n}
\end{array}\right)=\left(\begin{array}{c}
\mathbf{A} \mathbf{Q}^{n}+\boldsymbol{\mu}_{1}{ }^{n}+\boldsymbol{\mu}_{2}{ }^{n}+\mathbf{D} \\
-\mathbf{h}\left(\mathbf{Q}^{n}\right)+\mathbf{A}^{T} \mathbf{H}^{n}+\mathbf{B}^{T} \mathbf{H}^{f} \\
-\mathbf{g}^{F}\left(\boldsymbol{\mu}_{1}{ }^{n}\right)+\mathbf{H}^{n}-\mathbf{Z} \\
-\mathbf{g}^{V}\left(\boldsymbol{\mu}_{2}{ }^{n}\right)+\mathbf{H}^{n}-\mathbf{Z}
\end{array}\right)
$$

Where $\mathbf{F}_{n}=\nabla_{\boldsymbol{\eta}_{1}} \mathbf{g}^{F}\left(\boldsymbol{\mu}_{1}{ }^{n}\right)$ is the Jacobian matrix of the $\mathbf{g}^{F}$ function with regards to $\boldsymbol{\mu}_{1}$ at $\boldsymbol{\mu}_{1}{ }^{n}$; and $\mathbf{V}_{n}=\nabla_{\mathbf{\eta}_{2}} \mathbf{g}^{V}\left(\boldsymbol{\mu}_{2}{ }^{n}\right)$ is the Jacobian matrix of the $\mathbf{g}^{V}$ function with regards to $\boldsymbol{\mu}_{2}$ at $\boldsymbol{\mu}_{2}{ }^{n}$.

It reduced to the update formulae for the flow rate on the network graph and the leakage components: 


$$
\begin{gathered}
\mathbf{Q}^{n+1}=\mathbf{Q}^{n}-\mathbf{J}_{n}{ }^{-1}\left[\mathbf{h}\left(\mathbf{Q}^{n}\right)-\mathbf{A}^{T} \mathbf{H}^{n+1}-\mathbf{B}^{T} \mathbf{H}^{f}\right] \\
\boldsymbol{\mu}_{1}{ }^{n+1}=\boldsymbol{\mu}_{1}{ }^{n}-\mathbf{F}_{n}{ }^{-1}\left[\mathbf{g}^{F}\left(\boldsymbol{\mu}_{1}{ }^{n}\right)-\mathbf{H}^{n+1}+\mathbf{Z}\right] \\
\boldsymbol{\mu}_{2}{ }^{n+1}=\boldsymbol{\mu}_{2}{ }^{n}-\mathbf{V}_{n}{ }^{-1}\left[\mathbf{g}^{V}\left(\boldsymbol{\mu}_{2}{ }^{n}\right)-\mathbf{H}^{n+1}+\mathbf{Z}\right]
\end{gathered}
$$

Where $\mathbf{H}^{n+1}$ is solution of the linear system:

$$
\begin{gathered}
\left(\mathbf{A} \mathbf{J}_{n}{ }^{-1} \mathbf{A}^{T}+\mathbf{F}_{n}{ }^{-1}+\mathbf{V}_{n}{ }^{-1}\right) \mathbf{H}^{n+1}=\mathbf{A J}_{n}{ }^{-1}\left[\mathbf{h}\left(\mathbf{Q}^{n}\right)-\mathbf{B}^{T} \mathbf{H}^{f}\right]+\cdots \\
\mathbf{F}_{n}{ }^{-1}\left[\mathbf{g}^{F}\left(\boldsymbol{\mu}_{1}{ }^{n}\right)+\mathbf{Z}\right]+\mathbf{V}_{n}{ }^{-1}\left[\mathbf{g}^{V}\left(\boldsymbol{\mu}_{2}{ }^{n}\right)+\mathbf{Z}\right]-\left[\mathbf{A} \mathbf{Q}^{n}+\boldsymbol{\mu}_{1}{ }^{n}+\boldsymbol{\mu}_{2}{ }^{n}+\mathbf{D}\right]
\end{gathered}
$$

\section{Uniqueness and convergence properties}

The existence, uniqueness of solution and convergence properties of the power and modified orifice formulations may be investigated by introducing the minimization problem equivalent to system (16):

$$
\begin{aligned}
& \min C\left(\mathbf{Q}, \boldsymbol{\mu}_{1}, \boldsymbol{\mu}_{2}\right):=\sum_{j=1}^{n p} C_{j}\left(Q_{j}\right)+\sum_{i=1}^{n u} C_{i}^{F}\left(\mu_{1 i}\right)+\sum_{i=1}^{n u} C_{i}^{V}\left(\mu_{2 i}\right) \\
& \text { subject to: }-\sum_{j=1}^{n p} A_{i j} Q_{j}-\mu_{1 i}-\mu_{2 i}=D_{i}, \quad i=1, \cdots, n u
\end{aligned}
$$

Where $C$ is the Content function, a generalization of the Content function introduced by Piller et al. in 2003 for PDM (pressure driven modeling) and DDM (demand driven modeling) cases; $C_{j}\left(Q_{j}\right)=\int_{0}^{Q_{j}}\left[h_{j}(u)-\sum_{i=1}^{n f} B_{i j} H_{i}^{f}\right] d u$ is the contribution of link $\mathrm{j} ; C_{i}^{F}\left(\mu_{1 i}\right)=\int_{0}^{\mu_{1 i}}\left[g_{i}^{F}(\eta)+z_{i}\right] d \eta$ is the contribution for the fixed-area fictitious link at node $\mathrm{i}$; and $C_{i}^{V}\left(\mu_{2 i}\right)=\int_{0}^{\mu_{2 i}}\left[g_{i}^{V}(\eta)+z_{i}\right] d \eta$ is the contribution for the variablearea fictitious link at node i. $C$ has the dimension of power and is expressed with unknown flow rates and leakage components as basic unknowns. The gradient vector of the Content function has components:

$$
\begin{aligned}
& \frac{\partial C}{\partial Q_{j}}\left(\mathbf{Q}, \boldsymbol{\mu}_{1}, \boldsymbol{\mu}_{2}\right)=h_{j}\left(Q_{j}\right)-\sum_{i=1}^{n f} B_{i j} H_{i}^{f}, j=1, \cdots, n p \\
& \frac{\partial C}{\partial \mu_{1 i}}\left(\mathbf{Q}, \boldsymbol{\mu}_{1}, \boldsymbol{\mu}_{2}\right)=g_{i}^{F}\left(\mu_{1 i}\right)+z_{i}, \quad i=1, \cdots, n u \\
& \frac{\partial C}{\partial \mu_{2 i}}\left(\mathbf{Q}, \boldsymbol{\mu}_{1}, \boldsymbol{\mu}_{2}\right)=g_{i}^{V}\left(\mu_{2 i}\right)+z_{i}, \quad i=1, \cdots, n u
\end{aligned}
$$


And the Hessian matrix is diagonal with positive diagonal elements:

$\frac{\partial^{2} C}{\partial Q_{j}^{2}}\left(\mathbf{Q}, \boldsymbol{\mu}_{1}, \boldsymbol{\mu}_{2}\right)=\mathrm{J}_{j j}\left(Q_{j}\right) \geq 0, j=1, \cdots, n p$

$\frac{\partial^{2} C}{\partial \mu_{1 i}{ }^{2}}\left(\mathbf{Q}, \boldsymbol{\mu}_{1}, \boldsymbol{\mu}_{2}\right)=2 c_{1 i}{ }^{-2}\left\lfloor\mu_{1 i}\right\rfloor+\frac{h_{0}}{\Delta Q^{2}} \max \left(0,-\mu_{1 i}\right)=F_{i i}\left(\mu_{1 i}\right) \geq 0, \quad i=1, \cdots, n u$

$\frac{\partial^{2} C}{\partial \mu_{2 i}{ }^{2}}\left(\mathbf{Q}, \boldsymbol{\mu}_{1}, \boldsymbol{\mu}_{2}\right)=\frac{2}{3} c_{2 i}{ }^{-\frac{2}{3}}\left\lfloor\mu_{2 i}\right\rfloor^{-\frac{1}{3}}+\frac{h_{0}}{\Delta Q^{2}} \max \left(0,-\mu_{2 i}\right)=V_{i i}\left(\mu_{2 i}\right) \geq 0, \mu_{2 i} \neq 0, i=1, \cdots, n u$

The Hessian matrix is not defined for $\mu_{2 i}=0$ but this could be regularized around zero (Piller 1995).

$C$ is a strictly convex function because $h_{j}, g_{i}^{F}$ and $g_{i}^{V}$ are strictly increasing functions. This with the fact that the constraint set is convex proves the uniqueness of the solution. Moreover, $C$ is a norm-coercive function (it tends to plus infinity, if one of the component tend to plus or minus infinity). This means that level sets are bounded. The constraint set is nonempty and closed; $C$ is continuous and norm-coercive involve the existence of a solution for problem optimization (20).

Eq. (16) is the sufficient Euler-Lagrange condition for optimality of the Content model [Eq. (20)] (the nodal head is the Lagrange multiplier associated to the massbalance constraint). By convexity of $\mathrm{C}$ and full rank of the incidence matrix A solving the minimization problem (20) or the saddle point problem (16) are equivalent.

\section{Limitations of the formulations}

An important point to highlight is that there is no guarantee of global convergence (safe convergence whatever the initial point), even if Eq. (16) is stationary condition for a convex minimization problem (minimization of the Content). The Newton procedure can be unstable and the introduction of a damping (or under relaxation) factor has been proposed to get the global convergence under some conditions (Ortega 
and Rheinboldt, 1970). Non-convergence cases may occur in Epanet with emitter exponent greater or equal to two: the corresponding head loss function on the fictitious pipe is sub-linear and the first derivative of the head loss function may not be defined at the vicinity of the solution. For power equations with exponent between one and two, the number of iterations generally increases with increasing exponent, but it does converge. Similar reasoning can be done when comparing the GGA algorithm that is using head loss functions with exponents close to two and the Newton method applied to nodal equations that are using the inverse head functions with exponent close to 0.5 . The GGA algorithm is preferable because of producing less iteration in general. So, these limitations are common to all hydraulic solvers but depend on the formulation used.

Another known problem in Epanet is linked with presence of zero-flow conditions ( $\mathbf{J}_{n}$ is no longer invertible for zero flows or the ratio of the smallest to the largest elements on the diagonal of $\mathbf{J}_{n}$ is very large). Elhay and Simpson (2011) suggested a regularization technique that limits the bounds of the Schur complement eigenvalues. Only a modest extra computational cost is incurred when the technique is applied with a sufficient large bounded range. Other techniques may also be applied such as damping (plus a positive coefficient) on the head-loss Jacobian by Carpentier et al., (1987) or a modification of the Hazen-Williams friction head loss function near zero to force the slope to be greater than a fixed suitable number (Piller, 1995).

With the iterates (18) and (19), the first limitation is corrected as improved GGA is used with pipe head loss with exponent close to 2 and two emitters with exponent 0.5 and 1.5 , producing head loss on fictitious pipe of exponent 2 and $2 / 3$. For correcting the second problem of zero-flows in Epanet or other hydraulic solver, one of the techniques described before in the previous paragraph may be used (e.g. Piller, 1995). 


\section{APPLICATION TO EXAMPLE NETWORKS}

\section{Introduction}

The modified orifice model was implemented in the public domain Epanet hydraulic modeling package, and the implications of the modification tested on three networks of different sizes with stochastic leak distributions. The aim of this part of the study was to compare the proposed modified orifice leakage model with the conventional power leakage model in terms of simulation accuracy and convergence. This was done for the normal diurnal demand patterns, as well as cases where the system pressure was significantly reduced to investigate the model performance under pressure management conditions.

The modified orifice formulation was implemented by modifying the Epanet source code in such a way that each node can have two emitters and fixing the two emitter exponents to 0.5 and 1.5 respectively.

\section{Example networks}

Three networks of different sizes (small, medium and large) were used to evaluate the modified orifice formulation. The small and medium networks were adapted from Epanet's example networks Net1 and Net3 respectively, while the large network was adapted from the model of the central business district of Durban, a coastal city in South Africa.

Modifications were made to the example networks to ensure that they operate under gravity and each was supplied from a single point. Even though the example networks were modified, it should be noted that the formulations can be used to model networks with pumps and multiple sources. The purpose of the modifications was to 
control the pressures that were used in this study. Each network was simulated at two input pressures selected for the purposes of this study: a high initial pressure and a low pressure representing the implementation of pressure management to manage leakage. The difference between the initial and post-pressure management pressures were made intentionally large to allow simulation errors to be studied. These pressures are not unfeasible, but were not selected to represent typical distribution systems.

Table 1 summarizes the properties of the three example networks, including the average diurnal pressures at their average zonal points (AZPs), defined as the pressure at the node with an average static water pressure (AWWA 2016).

Table 1: System properties of the small, medium and large water distribution systems used in this study

\begin{tabular}{|l|c|c|l|c|c|}
\hline Network & $\begin{array}{l}\text { No of } \\
\text { Junctions }\end{array}$ & $\begin{array}{l}\text { Total pipe } \\
\text { length } \\
\mathbf{( k m )}\end{array}$ & $\begin{array}{l}\text { Pipe } \\
\text { diameter } \\
\text { range (mm) }\end{array}$ & $\begin{array}{l}\text { Initial } \\
\text { AZP (m) }\end{array}$ & $\begin{array}{l}\text { AZP after pressure } \\
\text { management (m) }\end{array}$ \\
\hline Small & 8 & 19.3 & $150-450$ & 81.2 & 21.8 \\
\hline Medium & 85 & 60.0 & $200-750$ & 86.7 & 37.6 \\
\hline Large & 747 & 103.8 & $25-800$ & 107.8 & 28.3 \\
\hline
\end{tabular}




\section{Leakage generation and distribution}

To implement realistic leakage distributions, leaks were generated and allocated to system nodes according to a stochastic model proposed by Schwaller and van Zyl (2014). One leak was generated at a time based on the statistical distributions described in Schwaller and van Zyl (2014), and summarized as follows:

- The discharge coefficient $C_{d}$ was modeled using a normal distribution.

- The initial leak area $A_{0}$ was modeled using a lognormal distribution for background leaks and a normal distribution for potentially detectable leaks.

- The head-area slope was calculated using a generalized power function of the initial leakage area based on work by Cassa \& Van Zyl (2013).

The generated leak was then allocated to a random pipe using a uniform distribution weighted by pipe length. Finally, a random number $R$ between zero and one was generated and used to distribute the leakage parameters between the two nodes of the pipe. Based on Eq. (3), the terms $R C_{d} A_{0} \sqrt{2 g}$ and $(1-R) C_{d} A_{0} \sqrt{2 g}$ were added to the coefficients of the emitter with an exponent of 0.5 of the two pipe nodes respectively. Similarly, the terms $R C_{d} m \sqrt{2 g}$ and $(1-R) C_{d} m \sqrt{2 g}$ were added to the coefficients of the emitter with an exponent of 1.5 of the two pipe nodes respectively.

Background leaks were generated first up to a level of $69 \%$ of the system's UARL as per the original definition of the UARL concept (Lambert, 2009). To replicate realistic systems where the leakage exponent of background leakage is often found to be close to 1.5 (Lambert, 2000; Lambert et al, 2013), $20 \%$ of the background leaks were assigned a head-area slope equivalent to a leakage number of 100, as was done by Schwaller and van Zyl (2014). 
Potentially detectable leaks were then generated up to the desired level of leakage for the system. In this study, the level of leakage used was at ILI of eight, which represents a system with high but not excessive leakage. Leaks were generated until the ILI was within $0.001 \%$ of the target value.

For the small network, $100 \%$ of the nodes had leakage, whereas for the medium and large networks, about $90 \%$ and $70 \%$ of the nodes, respectively, had leakage.

Once all the leaks were generated, the equivalent leakage parameters for a system modeled with the conventional power leakage approach were estimated. The same system nodes with leakage in the modified orifice approach were maintained in the conventional power leakage approach. To do this, two simulations were performed at different supply pressures (a $5 \mathrm{~m}$ head differential was used) under minimum night flow conditions. Two average zonal night pressures and corresponding system leakage flows were then obtained and used to estimate the system leakage exponent. Once the leakage exponent was known, the leakage coefficients for identical nodal leakage flows were calculated for each node under minimum night flow conditions.

The above process was used to generate in total 600 random leakage distribution scenarios, whereby each of the three example networks had 200 scenarios, each with an ILI of eight.

\section{RESULTS}

The 200 leakage scenarios in each of the three networks were simulated using both the conventional power equation and the newly implemented modified orifice approaches. The results were then analyzed to allow the performance of the two algorithms to be compared both in terms of simulation accuracy and convergence speed. 
It should be noted that, the modified orifice equation is based on a fundamental fluid mechanics theory and incorporating the linear head-area slope, that was demonstrated in several studies under elastic and viscoelastic deformation conditions. In contrast, the power equation is purely an empirical equation with no theoretical basis. Thus, when presenting the results, the modified orifice formulation was assumed to provide the true behavior and differences between the results of the two formulations were described as errors in the power equation formulation.

\section{Simulation accuracy}

Figure 1 shows the variation of the medium system's pressure at its average zonal point without leakage and under initial and pressure management conditions for both formulations. The pressure for the medium system without any leakage is also shown and even though the system had a high level of leakage, it had only a small influence on the average zonal pressure.

Due to the small influence of leakage on pressure, no differences in the pressures obtained from the modified orifice and power formulations are evident in the figure.

The volumetric error in the total daily leakage volumes (the fractional difference in total system leakage volume over a 24-hour simulation) for the two leakage formulations is negligibly small for all three systems without pressure management as shown in figure 2.

However, after pressure management the power formulation error in the daily leakage volume becomes substantial as shown in figure 3. The power formulation is an empirical approach that can only be used safely within its pressure calibration range. Since the power formulation in the example was calibrated under minimum night flow conditions for the initial system, it is not able to accurately model system leakage at 
the substantially different pressures resulting from pressure management. This is an important factor to consider when estimating the expected savings in leakage when planning pressure management interventions.

Finally, the results showed that while the error in total system leakage for the systems without pressure management was negligible (figure 2), this was not true for the leakage volumes at individual nodes. Figure 4 shows the typical leakage pattern at the critical node (system node with the lowest pressure) of the medium system for the two formulations, both with and without pressure management.

The two formulations give identical leakage in the initial system under minimum night flow conditions, since this is where the power formulation was calibrated. However, substantial differences are evident for other times in the initial system and for the whole day during pressure management.

\section{Convergence}

The modified orifice formulation required slightly more iterations to converge compared to the power formulation for all three systems as shown in figure 5 . This increase is likely due to increased number of unknowns in the modified orifice equation, as a result of adding a second emitter to each node.

It is known that the global gradient method has convergence problems under certain conditions. Some of these problems will be experienced with both the power and modified orifice formulations, for instance due to ill-conditioned Jacobian matrices, badly chosen initial solutions, a large range of link resistances or a lack of smoothness in the head loss function or its derivatives (inverse of a leakage function is like a head loss function). 
However, it was found for the power formulation that an equivalent leakage exponent greater than two sometimes resulted in non-convergence of the standard global gradient algorithm. Since the modified orifice exponents are fixed at 0.5 and 1.5 , this problem cannot be experienced by this formulation.

For the power equation formulation, leakage exponents above two sometimes occurred when generating stochastic systems with high ILI values. These large leakage exponents are not unrealistic and have been observed in field and laboratory studies (Greyvenstein, 2007; see Schwaller et al. (2014) for a summary of international field studies), and thus this problem needs to be addressed.

The reason for the non-convergence was found to be related to conditions under which the Newton Raphson method overshoots the root and subsequently diverges away from it. In the global gradient algorithm, the emitter behavior is simulated through the head loss function of a fictional pipe, which is a power function of flow rate with an exponent of $1 / \alpha$. Non-convergence of this function for $\alpha>2$ is well known (for instance see Wikipedia, 2016).

Like most other convergence problems in the global gradient algorithm, this problem may be solved by introducing a damping parameter into the algorithm.

\section{CONCLUSION}

This study investigated the implementation of a modified orifice formulation for more realistic simulation of leakage in water distribution systems. A modified hydraulic network formulation is proposed that includes a second emitter at each leakage node.

Application of the modified formulation showed that the power and modified orifice leakage formulations produced similar results for total system leakage flows and 
volumes under normal diurnal pressure variations. However, the leakage flows at individual nodes at elevations different from the average zonal pressure were found to differ significantly.

In addition, it was found that simulating systems at pressures significantly different from the normal diurnal range, for instance as a result of pressure management, results in substantial errors in both leakage flows and volumes.

The average convergence speed of the modified orifice formulation was found to be slightly larger than that of the power formulation with, on average, one more iterations required.

The current power formulation used in the global gradient algorithm (and implemented in Epanet) resulted in non-convergence when a leakage exponent greater than two was used. Like most other known instabilities of the Newton-Raphson method, this problem can be solved by introducing a damping factor into the convergence process.

Finally, further studies are recommended to investigate the implications of a more realistic modified orifice leakage formulation on the results of modeling studies that include leakage, such as calibration, leak detection and operational optimization studies.

\section{ACKNOWLEDGEMENT}

The authors would like to express their appreciation to Mr. Simon Scruton and his team at the eThekwini Water and Sanitation unit, for providing water distribution network model of the central business district of Durban, from which the large network was adapted. 


\section{REFERENCES}

AWWA (2016) M36 - Water audits and loss control programs, Fourth Edition, American Water Works Association.

Carpentier, P., Cohen, G., and Hamam, Y. (1985). "Water Network Equilibrium, Variational Formulation and Comparison of Numerical Algorithms." EURO VII, Proc. 7th European Congress on Operational Research, Bologna, IT, printed by Bologna Tecnoprint, 1985, 30.

Cassa, A.M., van Zyl, J.E., Laubscher, R.F. (2010) A numerical investigation into the effect of pressure on holes and cracks in water supply pipes, Urban Water Journal, 7 (2) $109-120$.

Cassa, A.M., van Zyl, J.E. (2013) Predicting the pressure-leakage slope of cracks in pipes subject to elastic deformations, Journal of Water Supply: Research and Technology - AQUA, 62 (4) 214-223.

De Marchis, M., Fontanazza, C.M., Freni, G., Notaro, V., Puleo, V. (2016) Experimental Evidence of Leaks in Elastic Pipes, Water Resources Management 30:2005-2019

Elhay, S., and Simpson, A. (2011). "Dealing with Zero Flows in Solving the Nonlinear Equations for Water Distribution Systems." Journal of Hydraulic Engineering, 137(10), 1216-1224.

Elhay, S., Piller, O., Deuerlein, J., and Simpson, A. (2015). "A Robust, Rapidly Convergent Method That Solves the Water Distribution Equations for PressureDependent Models." Journal of Water Resources Planning and Management, 0(0), 04015047. 
Farley, M. \& Trow, S., 2003. Losses in Water Distribution Network. London: IWA Publishing.

Ferrante, M., Massari, C., Brunone, B. \& Meniconi, S., 2011. Experimental Evidence of Hysteresis in the Head-Discharge Relationship for a Leak in a Polyethylene Pipe. Journal of Hydraulic Engineering, Issue 137-7, pp. 775-780.

Fox, S., Collins, R., Boxall, J. (2016) Experimental study exploring the interaction of structural and leakage dynamics, Journal of Hydraulic Engineering, 04016080.

Greyvenstein, B., Van Zyl, J.E. (2007). “An experimental investigation into the pressure-leakage relationship of some failed water pipes”, Journal of Water Supply: Research and Technology - AQUA, 56 (2) 117-124.

Hamam, Y. M., and Brameller, A. (1971). "Hybrid Method for the Solution of Piping Networks." IEE Proceedings, 118(11).

Hiki, S., 1981. Relationship between Leakage and Pressure. Japan Waterworks Association Journal, May, pp. 50-54.

Lambert A. 2000 What Do We Know About Pressure: Leakage Relationship in Distribution System? In: System Approach to Leakage Control and Water Distribution Systems Management, Brno, Czech Republic.

Lambert A O, 2002 International Report: Water Losses Management and techniques. Journal of Water Science and Technology: Water Supply Vol 2, Issue 4, pp 1-20

Lambert, A. 2009 Ten years' experience in using the UARL formula to calculate Infrastructure Leakage Index. IWA International Specialized Conference 'Water Loss 2009’. Conference Proceedings, April 2009, Cape Town, South Africa. 
Lambert, A., Fantozzi, M., and Thornton, J. (2013). "Practical approaches to modeling leakage and pressure management in distribution systems - Progress since 2005." Proc., 12th Int. Conf. on Computing and Control for the Water Industry, CCWI2013, Perugia, Italy.

May J. H. (1994) Leakage, Pressure and Control. In: Proceedings of the BICS International Conference Leakage Control Investing in Underground Assets, The SAS Portman Hotel.

Malde, R. (2015) An analysis of leakage parameters of individual leaks on a pressure pipeline through the development and application of a standard procedure, M.Sc. dissertation, University of Cape Town.

Massari, C., Ferrante, M., Brunone, B., Meniconi, S. (2012) Is the leak headdischarge relationship in polyethylene pipes a bijective function? Journal of Hydraulic Research 50 (2012) 409-417.

Ogura, 1979. Experiment on the Relationship between Leakage and Pressure. Japan Water Works Association, June, pp. 38-45.

Ortega, J. M., and Rheinboldt, W. C. (1970). "Chapter 14 - CONVERGENCE OF MINIMIZATION METHODS." Iterative Solution of Nonlinear Equations in Several Variables, Academic Press, 473-520.

Piller, O., Bremond, B., and Poulton, M. (2003). "Least Action Principles Appropriate to Pressure Driven Models of Pipe Networks". World Water and Environmental Resources Congress 2003 (EWRI03), 2003, Philadelphia (PA), US, printed by ASCE, pp. 1-15 (CD-ROM). 
Piller, O., and van Zyl, J. (2014). "Modeling Control Valves in Water Distribution Systems Using a Continuous State Formulation." Journal of Hydraulic Engineering, ASCE, 140(11), 04014052.

Rossman, L. A. (2000). Epanet 2 User's Manual, US Environmental Protection Agency, Cincinnati.

Schwaller, J., Van Zyl, J.E. (2014) Modeling the pressure-leakage response of water distribution systems based on individual leak behavior. Journal of Hydraulic Engineering, 04014089.

Schwaller, J., Van Zyl, J.E., Kabaasha, A.M. (2015) Characterising the pressureleakage response of pipe networks using the FAVAD equation, Water Science \& Technology: Water Supply, 15 (6) 1373 - 1382

Ssozi, E.N., Reddy, B.D., Van Zyl, J.E. (2016) Numerical investigation of the influence of viscoelastic deformation on the pressure-leakage behavior of plastic pipes, Journal of Hydraulic Engineering, 142 (3) DOI: 10.1061/(ASCE)HY.19437900.0001095 .

Todini, E., and Pilati, S. (1988). "A Gradient Projection Algorithm for the Analysis of Pipe Networks." Computer Applications for Water Supply and Distribution, Leicester Polytechnic, Research Study Press, Taunton, UK, Volume 1/1, 1-20.

Van Zyl, J.E., Alsaydalani, M.O.A., Clayton, C.R.I., Bird, T., \& Dennis, A. (2013). Soil fluidization outside leaks in water distribution pipes - Preliminary observations. Proceedings of the Institute of Civil Engineers. Water management. 166(10), 546-555. Van Zyl, J.E., Cassa, A.M. (2014) "Modeling elastically deforming leaks in water distribution pipes", Journal of Hydraulic Engineering, 140 (2) 182 - 189. 
Van Zyl, J.E., Clayton, C.R.I. (2007). "The effect of pressure on leakage in water distribution systems", Water Management, 160 (WM2) 109-114.

Van Zyl, J.E., Lambert, A.O., Collins, R. (in press) Realistic modeling of leakage and intrusion flows through leak openings in pipes, Journal of Hydraulic Engineering.

Wikipedia (2016) Newton's Method, https://en.wikipedia.org/wiki/Newton's_method (accessed 19 December 2016).

Walski, T., Bezts, W., Posluzny, E., Weir, M. and Whitman, B. (2006). Modelling Leakage Reduction through Pressure Control. Journal of the American Water Work Association, Vol. 98, no. 4, pp.147-152. 